\title{
Multiple Pathways Regulate the Induction of Genes During Zoosporogenesis in Phytophthora infestans
}

\author{
Shuji Tani, ${ }^{1}$ Einat Yatzkan, ${ }^{2}$ and Howard S. Judelson ${ }^{1}$ \\ ${ }^{1}$ Department of Plant Pathology, University of California, Riverside 92521, U.S.A.; ${ }^{2}$ Syngenta Biotechnology, Inc., 3054 \\ Cornwallis Road, Research Triangle Park, NC 27709, U.S.A.
}

Submitted 4 August 2003. Accepted 4 November 2003.

\begin{abstract}
Zoospores are a critical component of the disease cycles of most oomycete pathogens. To better understand this stage, genes induced during zoosporogenesis were identified from Phytophthora infestans, the potato late blight pathogen. Using cDNA arrays representing 2,600 genes expressed during zoosporogenesis, 69 genes showing >fourfold increases in mRNA levels were identified, of which 22 exhibited $>100$-fold induction. Included were putative protein kinases, transcription factors, ion channels, and other regulators. The expression of 15 genes was characterized in detail using zoosporogenesis time courses, other developmental stages, different temperature regimes, and tissue treated with signaling inhibitors. The latter were of interest because zoosporogenesis is known to be cold induced and inhibited by calcium channel blockers such as verapamil; moreover, in this study, inhibitors of phospholipase C (U-73122) and inositol trisphosphate receptor-gated calcium channels (2-aminoethoxydiphenyl borate) also were shown to block zoosporogenesis. The results indicated that the cytoplasmic and transcriptional changes occurring during zoosporogenesis are regulated by several pathways. For example, verapamil inhibited zoosporogenesis but not the up-regulation of most genes; the induction of some genes required while others were independent of calcium or phospholipid signaling; and, although most genes were induced in sporangia at $10^{\circ} \mathrm{C}$ but not $24^{\circ} \mathrm{C}$, one was induced at both temperatures.
\end{abstract}

Oomycetes, including Phytophthora and Pythium spp. and the downy mildews, cause devastating diseases of many plants. Contributing to the success of most of these pathogens is their ability to release numerous motile zoospores from previously multinucleate coenocytic sporangia. In Phytophthora infestans, for example, placing sporangia into cool water (usually less than $12^{\circ} \mathrm{C}$ ) for approximately $1 \mathrm{~h}$ induces cytoplasm to cleave into six or more biflagellated zoospores (Ribeiro 1983). At temperatures higher than $20^{\circ} \mathrm{C}$, it is more common for sporangia to produce a germ tube directly. Zoospores are thought to be responsible for most natural infections (Duniway 1983). After they emerge from sporangia, zoospores swim chemotactically and rapidly encyst in response to physical and chemical stimuli. This involves the detachment of flagella, secretion of the cyst wall, and release of an adhesive glyco-

Corresponding author: H. S. Judelson; Telephone: 909-787-4199; Fax: 909-787-4294; E-mail: howard.judelson@ucr.edu

Sequences are available in the GenBank database under accession numbers CF762005 to CF762011, CF774503 to CF774559, and CF798507 to CF798509. protein. Cysts subsequently produce a germ tube which develop an appressorium prior to host penetration (Hardham 2001).

Elegant cytological studies have examined the cleavage of sporangial cytoplasm, the release of zoospores, encystment, and cyst germination (Hardham 2001). Some structural components of the zoospore or cyst also have been identified (Marshall et al. 2001). However, little is known of the molecular basis of zoospore development. It generally is assumed that sporangia are preprogrammed to undergo zoosporogenesis, because actinomycin and cycloheximide do not block zoospore release (Clark et al. 1978; Penington et al. 1989). However, a recent study questioned whether such inhibitors enter sporangia fast enough to stop transcription during cleavage (Judelson and Roberts 2002). Although zoospores also are believed to be preprogrammed to encyst, germination of the cysts and subsequent appressorium development requires de novo RNA and protein synthesis (Clark et al. 1978; Penington et al. 1989).

Cations, especially calcium, are reported to regulate each phase of zoospore biology (Bircher and Hohl 1999; Griffith et al. 1988; Jackson and Hardham 1996). Such information comes mostly from inhibitor studies, although measurements of calcium levels also indicated that encystment involves the release and reabsorption of this ion (Warburton and Deacon 1998). Calcium flux during zoospore formation is not well understood, although its transfer from intracellular stores is implicated. How and where this happens in Phytophthora spp. is unknown, but such pathways are well described in other species. In most eukaryotes, calcium reserves are primarily within the endoplasmic reticulum and are transported into cytoplasm by various pumps and channels (Gaffin 1999). One well-conserved mechanism for this involves a channel regulated by a receptor for the secondary messenger inositol trisphosphate $\left(\mathrm{IP}_{3}\right)$, which is synthesized along with diacylglycerol by phospholipase C (Patel et al. 1999). Oscillations in $\mathrm{IP}_{3}$ concentrations are known to regulate a range of cellular responses (Bockaert and Pin 1999).

To augment our understanding of the biology of $P$. infestans zoospores, we have combined a study of genes induced during the initial phases of zoosporogenesis (i.e., the cleavage of sporangial cytoplasm) with an examination of the effects of inhibitors of calcium and $\mathrm{IP}_{3}$ signaling on development and transcription. In all, 69 genes showing large increases in mRNA abundance during cleavage were identified, of which several appear to be candidates for regulators of the zoospore pathway. Inhibitors of phospholipase $\mathrm{C}, \mathrm{IP}_{3}$-gated ion channels, and other calcium channels each blocked zoosporogenesis but had diverse effects on the expression of the genes induced during that stage. Differences between genes also were noted in their temporal pattern of expression and response to cool temperatures, suggesting 
that multiple signal transduction pathways regulate cellular events during zoosporogenesis.

\section{RESULTS}

\section{Genes induced during zoosporogenesis.}

Macroarrays spotted with inserts from a cDNA library of cleaving sporangia were used to identify genes up-regulated during zoosporogenesis. That library contained 4,174 clones, which later were estimated to represent 2,617 unique genes based on assemblies of $5^{\prime}$ single-pass sequence data. Two arrays representing each of these genes were hybridized to radiolabeled total cDNA from sporangia incubated in $10^{\circ} \mathrm{C}$ water to induce cleavage, and cDNA from undifferentiated sporangia. Up-regulated genes were identified after normalization to actin $(a c t A)$ and translation elongation factor- $1 \alpha(\mathrm{EF} 1)$. The up-regulated genes were confirmed using secondary and tertiary screens, each involving new arrays containing duplicate spots of each clone and cDNA probes from tissue prepared independently from that of the prior screens. Representative portions of the tertiary array are shown in Figure 1A.

After the tertiary screen, redundancy among the up-regulated clones was identified using data from $5^{\prime}$ and $3^{\prime}$ DNA sequence reads, which defined 69 nonredundant genes with $P$. infestans cleavage (pic) loci (Table 1). These were selected to fit the following criteria: >fourfold induction during cleavage, as calculated from the tertiary screen; consistent up-regulation in the primary, secondary, and tertiary screens; signal-to-noise ratios with cleaving probes of $>5.0$; and induction significant at $P<0.05$ based on $t$-test analysis. To confirm that spots representing the same gene on different array filters contained the same amount of DNA, one of the polymerase chain reaction (PCR) primers that had been used to amplify the DNA for array construction was radiolabeled and hybridized to the terti-
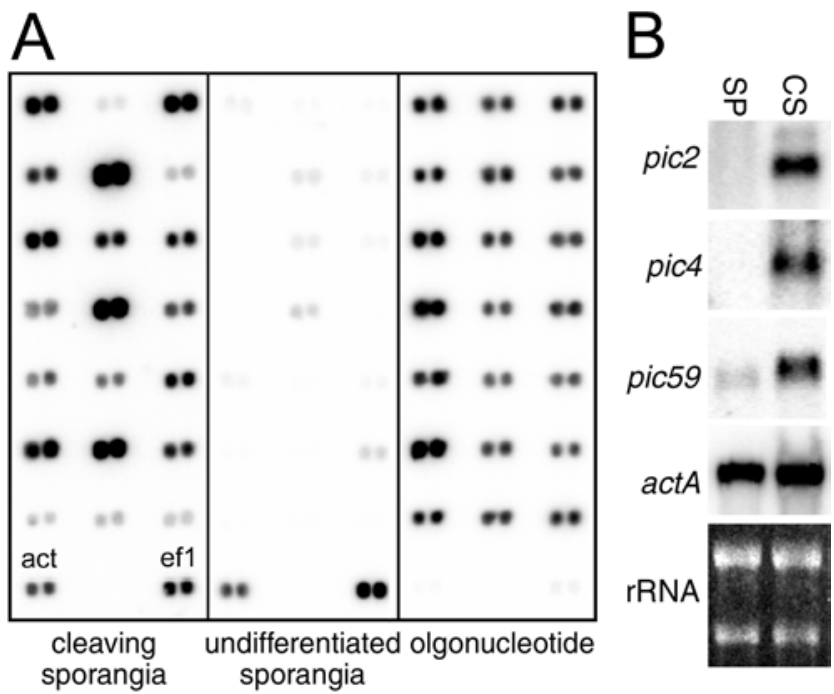

Fig. 1. Assays for cleavage-induced genes. A, Representative portion of array filter used in tertiary screen. Spots in seven top rows represent inserts from 21 clones from cleaving cDNA library (in duplicate), and those in lower row represent actin (act) and elongation factor-1 $\alpha$ (ef1) normalization controls. The left, middle, and right panels represent hybridizations with ${ }^{32} \mathrm{P}$-cDNA from cleaving and undifferentiated sporangia, and a ${ }^{32} \mathrm{P}$-labeled oligonucleotide that binds to the $5^{\prime}$ end of each DNA fragment. Much less actin and elongation factor-1 $\alpha$ DNA was intentionally spotted on the arrays; although their signals using the oligonucleotide probe appear faint, they were within the linear range of the phosphorimager. B, Blot of RNA from undifferentiated and cleaving sporangia (SP and CS, respectively), hybridized with three pic genes or an actin $(a c t A)$ control. An ethidium bromide-stained profile of rRNA in the gels is shown at the base of the figure as a loading control. ary arrays. This showed that the replicates contained equivalent amounts of DNA $( \pm 6 \%)$.

RNA blot analysis of 18 pic genes confirmed that each was up-regulated during cleavage. This is shown in Figure 1B for three genes using blots of RNA from undifferentiated and cleaving sporangia. Similar confirmatory data for 15 other pic genes are presented later in this article (Figs. 2 and 3), as part of more detailed analyses of their expression.

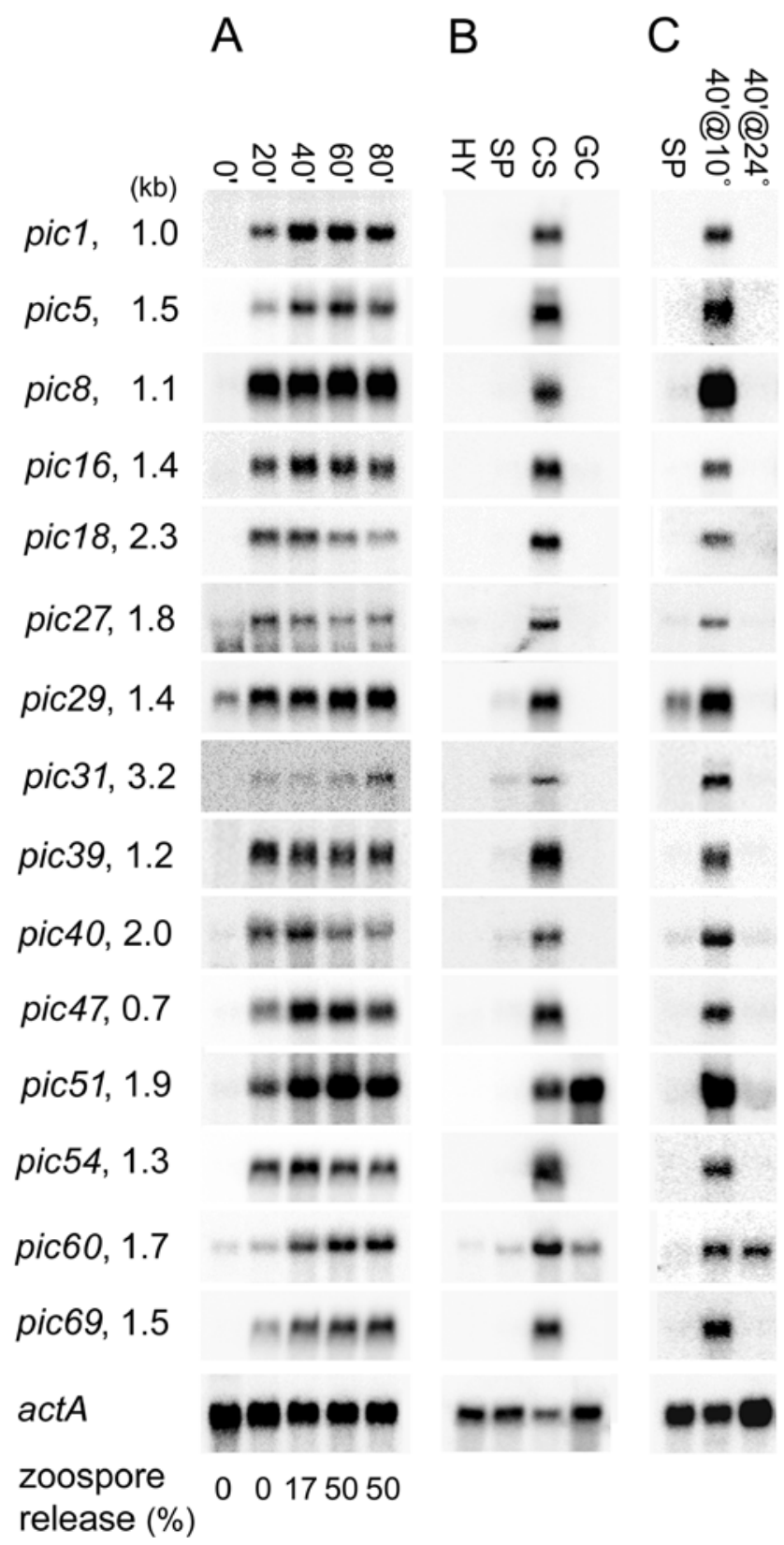

Fig. 2. Expression of Phytophthora infestans genes induced during zoosporogenesis. Total RNA was electrophoresed, blotted, and hybridized with a probe for the indicated pic genes. Blots then were reprobed with an actin $(a c t A)$ clone. One representative actin hybridization is shown; other blots gave equivalent results. A, RNA from freshly harvested sporangia $\left(0^{\prime}\right)$ and sporangia kept at $10^{\circ} \mathrm{C}$ for 20 to $80 \mathrm{~min}$ to induce cleavage. The percentage of sporangia releasing zoospores at each timepoint is displayed at the base of the panel; when zoospores were present, the zoospores and sporangia were combined in the RNA extractions. B, RNA from nonsporulating hyphae (HY), freshly harvested sporangia (SP), sporangia incubated at $10^{\circ} \mathrm{C}$ for $40 \mathrm{~min}$ to induce cleavage (CS), and cysts germinated in water for $6 \mathrm{~h}(\mathrm{GC})$. C, RNA from freshly harvested sporangia (SP), sporangia kept for $40 \mathrm{~min}$ at $10^{\circ} \mathrm{C}\left(40^{\prime} @ 10^{\circ}\right)$ or at $24^{\circ} \mathrm{C}$ $\left(40^{\prime} @ 24^{\circ}\right)$. 
A

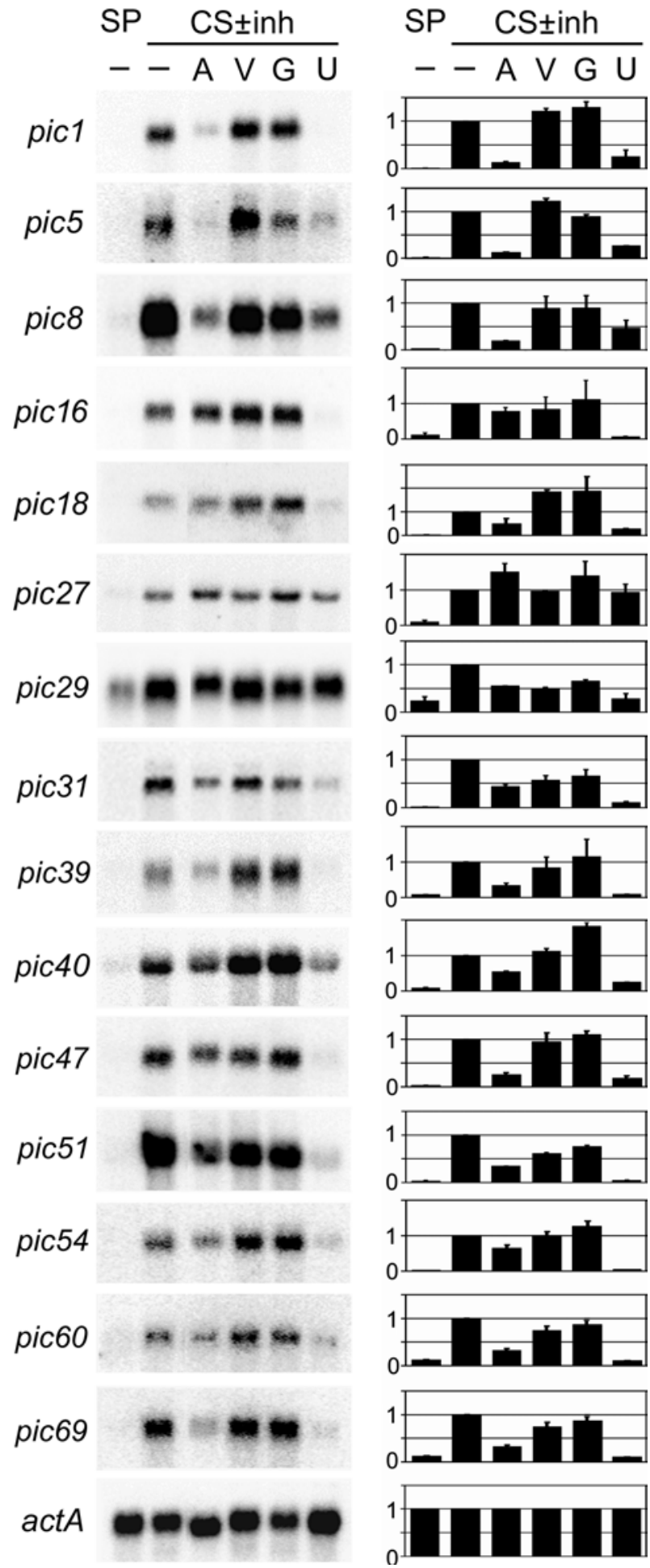

Fig. 3. Effect of inhibitors on mRNA accumulation. Analyzed were freshly harvested sporangia (SP), or sporangia kept at $10^{\circ} \mathrm{C}$ for $40 \mathrm{~min}$ to induce cleavage, with or without inhibitors ( $\mathrm{CS} \pm$ inh). The latter were treated with no inhibitor (-), $10 \mu \mathrm{M}$ 2-aminoethoxydiphenyl borate (A), $200 \mu \mathrm{M}$ verapamil (V), $10 \mu \mathrm{M}$ gadolinium (G), or $1 \mu \mathrm{M}$ U-73122 (U). In the absence of inhibitors, approximately $20 \%$ of sporangia held at $10^{\circ} \mathrm{C}$ would have released zoospores. A, RNA blots hybridized with probes for pic or actA genes. The experiment was performed twice using RNA prepared on different days, but only one study is shown. Each filter was reprobed with actA but only one representative blot is shown. B, Quantification of blots shown in A. Signals were measured by phosphorimager analysis, normalized to actin, and standardized such that a value of 1 equals the signal from cleaving sporangia without inhibitors (CS, - ). Data are averages of the two independent experiments \pm range.
The abundance of mRNA from each gene during cleavage, relative to the level of EF1, was calculated (Table 1). This entailed dividing the signal from the ${ }^{32} \mathrm{P}$-labeled cDNA probe from cleaving sporangia by the signal from the oligonucleotide, followed by normalization to EF1. The abundance of the pic mRNAs ranged from 1 to $23 \%$ of that of EF1, with a median of $4 \%$. Based on the abundance of EF1 sequences within the cleaving sporangia cDNA library, the 69 induced genes are estimated to represent $0.9 \%$ of total mRNA.

\section{Sequence analysis.}

An average of 950 nucleotides (nt) of sequence was obtained for each gene by sequencing from the $5^{\prime}$ and $3^{\prime}$ ends of a differentially expressed cDNA clone and, in some cases, by analyzing cross-hybridizing clones from a cDNA library. By comparison, a typical full-length $P$. infestans mRNA can be predicted from sequences in a public expressed sequence tag (EST) database to be approximately 1,150 nt (Kamoun et al. 1999). Full-length open reading frames could be predicted for 42 of the 69 pic genes. A comparison of the sequences with GenBank revealed matches for 28 of the 69 genes $(41 \%)$, using an $E$-value cutoff of $10^{-5}$ (Table 1$)$. Only pic16 and pic51 matched known Phytophthora genes (Fabritius and Judelson 2003; Judelson and Roberts 2002). The fraction of matches to genes in other species was lower than in prior studies, suggesting that many genes induced during zoosporogenesis have novel functions. For example, 53 of 61 sporulationinduced sequences (87\%) having an average contig size of 897 nt resembled entries in GenBank (Kim and Judelson 2003). Among random ESTs having an average size of $586 \mathrm{nt}, 63 \%$ showed matches (Kamoun et al. 1999). Of the genes having informative matches in GenBank, one appeared to participate in transcriptional regulation, four in protein phosphorylation, five in transporting small molecules, six in metabolism, and two in protein degradation.

Expression during cleavage and other developmental stages.

RNA blot analysis was used to better characterize pic expression and further confirm the array data. Fifteen clones with interesting matches in GenBank plus those representative of high and moderate induction ratios were hybridized to RNA from sporangia placed in $10^{\circ} \mathrm{C}$ water for 0 to $80 \mathrm{~min}$ to stimulate cleavage (Fig. 2A); hyphae, sporangia, cleaving sporangia, and 6-h-germinated zoospore cysts (Fig. 2B); and sporangia placed for $40 \mathrm{~min}$ in $10^{\circ} \mathrm{C}$ water to induce cleavage, or at $24^{\circ} \mathrm{C}$ to induce direct germination (Fig. $2 \mathrm{C}$ ). During the $10^{\circ} \mathrm{C}$ treatments, zoospore release typically began by $40 \mathrm{~min}$ and plateaued by 60 to $80 \mathrm{~min}$. In the experiment shown, approximately $50 \%$ of sporangia released zoospores, which is typical for the strain of $P$. infestans employed.

In the cleavage time course, all 15 genes were induced within $20 \mathrm{~min}$. However, at least four patterns of expression were noted (Fig. 2A). The most common profile was characterized by mRNAs that, by $40 \mathrm{~min}$, reached their maximum levels, which were maintained through $80 \mathrm{~min}$ (pic1, 5, 16, 51, 60, and 69). A second group of genes showed a similar pattern but attained peak mRNA abundance faster (pic8, 29, and 39). Another pattern of expression involved the slow accumulation of mRNA, which peaked by $80 \mathrm{~min}$ (pic 31). In a fourth pattern, mRNAs levels peaked by 20 to $40 \mathrm{~min}$ and then decayed (pic18, 27, 40, 47, and 54). This suggests that the genes are regulated by several distinct mechanisms, although the relative contributions of transcription and degradation cannot be assessed.

Although this RNA blot experiment confirmed that the genes identified using the cDNA arrays were up-regulated during cleavage, the degree of induction of some genes appeared 
higher in the blots than the arrays. For example, pic69 measured fourfold induction in the arrays but 11-fold in the RNA blot. This may be due to differences in the tissues used for each experiment, or technical considerations. Array studies frequently underestimate the level of induction of a gene, due to nonspecific hybridization resulting from gene families or other factors (Taniguchi et al. 2001).

Analyses of different developmental stages of $P$. infestans suggested that most, but not all, genes were expressed specifically during cleavage (Fig. 2B). One exception was pic51, which was expressed more in germinated cysts than in cleaving sporangia. A probe for pic60 also detected a signal in germinated cysts, although it was lower than in cleaving sporangia. The strong expression of pic51 at a stage during which plant interactions occur was notable because this gene encodes an elicitor of defense responses in potato and parsley, which are hosts and nonhosts, respectively, of $P$. infestans (Brunner et al. 2002; Fabritius and Judelson 2003).

For several genes (pic29, 31, and 40), a weak signal was detected in freshly harvested sporangia, and this may be interpreted in two ways. Such genes may be expressed in sporangia at a low level, prior to experiencing a massive induction during cleavage. Alternatively, this simply may reflect the fact that a true "zero-timepoint" sample cannot be obtained, because approximately $5 \mathrm{~min}$ are required to harvest sporangia; during this time, some induction of pic genes may occur. Attempts to resolve this by harvesting sporangia in solutions containing actinomycin D were unsuccessful, probably because this in- hibitor does not enter sporangia swiftly enough to completely block transcription during cleavage (Judelson and Roberts 2002).

Probes for some genes, such as pic60, detected weak signals in hyphal RNA (Fig. 2B, HY). This raised the possibility that some of the pic genes might not be best described as being specific to zoosporogenesis; instead, they conceivably might be expressed in hyphae, down-regulated in sporangia, and reactivated once sporangia began to germinate as part of their return to hyphal growth. To test this, the cDNA macroarrays were hybridized with a hyphal cDNA probe (Table 1). For the vast majority of pic genes, expression appeared specific to cleaving sporangia because mRNA levels in hyphae were very low, usually similar to their level in sporangia.

All but 1 of the 15 genes tested in the RNA blots were specifically induced by immersing sporangia for $40 \mathrm{~min}$ in 10 versus $24^{\circ} \mathrm{C}$ water (Fig. 2C). As previously described (Ribeiro 1983), while the $10^{\circ} \mathrm{C}$ treatment induces zoospores, direct germination is enabled at $24^{\circ} \mathrm{C}$ (in the experiment shown, $>70 \%$ of sporangia generated direct germ tubes at $24^{\circ} \mathrm{C}$ while none released zoospores). This suggests that exposure to cool water, as opposed to immersion in water itself, specifically stimulates both zoosporogenesis and the expression of most pic genes. The one exception was pic60, which also had the uncommon characteristic of being expressed in germinated cysts. Both treatments resulting in pic60 expression involved immersing tissue in water; therefore, it is possible that a condition such as nutrient limitation is the primary inducer of this gene.

Table 1. Phytophthora infestans genes induced $>$ fourfold during zoosporogenesis

\begin{tabular}{|c|c|c|c|c|c|c|c|}
\hline \multirow[b]{2}{*}{ Gene } & \multirow[b]{2}{*}{ Number $^{b}$} & \multicolumn{2}{|c|}{ mRNA level in CS vs. ${ }^{a}$} & \multirow{2}{*}{$\frac{\text { Hyphae vs. }}{\text { CS }^{\text {d }}}$} & \multicolumn{3}{|c|}{ Best hit in GenBank } \\
\hline & & Sporangia $^{c}$ & EF-1 & & Number $^{\mathrm{b}}$ & Gene description, source species & $E$ value $^{\mathrm{e}}$ \\
\hline picl & CF7744503 & $>150$ & 0.01 & $0.00 \pm 0.00$ & AF441288 & Nuclear LIM factor interactor, Xenopus laevis & $1 \mathrm{E}-50$ \\
\hline pic2 & CF7744504 & $>150$ & 0.04 & $0.00 \pm 0.00$ & No hit & $\ldots$ & $\ldots$ \\
\hline pic3 & CF7744505 & $>150$ & 0.09 & $0.00 \pm 0.00$ & No hit & $\ldots$ & $\ldots$ \\
\hline pic4 & CF7744506 & $>150$ & 0.05 & $0.00 \pm 0.00$ & No hit & $\ldots$ & $\ldots$ \\
\hline pic5 & CF7744507 & $>150$ & 0.08 & $0.00 \pm 0.00$ & AY133763 & Adenylate kinase, Arabidopsis thaliana & $2 \mathrm{E}-33$ \\
\hline pic6 & CF7744508 & $>150$ & 0.08 & $0.00 \pm 0.00$ & NP081295 & Sperm tail protein, Mus musculus & $4 \mathrm{E}-07$ \\
\hline pic7 & CF762005 & $>150$ & 0.02 & $0.00 \pm 0.00$ & No hit & $\ldots$ & $\ldots$ \\
\hline pic8 & CF7744509 & $>150$ & 0.01 & $0.00 \pm 0.00$ & No hit & $\ldots$ & $\ldots$ \\
\hline pic 9 & CF7744510 & $>150$ & 0.12 & $0.00 \pm 0.00$ & NP035787 & Tubby-like protein, M. musculus & $3 \mathrm{E}-12$ \\
\hline pic10 & CF7744511 & $>150$ & 0.08 & $0.00 \pm 0.00$ & No hit & $\ldots$ & $\ldots$ \\
\hline pic11 & CF7744512 & $>150$ & 0.03 & $0.01 \pm 0.01 *$ & No hit & $\ldots$ & $\ldots$ \\
\hline pic12 & CF762006 & $>150$ & 0.03 & $0.00 \pm 0.00$ & No hit & $\ldots$ & $\ldots$ \\
\hline pic13 & CF7744513 & $>150$ & 0.20 & $0.00 \pm 0.00$ & No hit & $\ldots$ & $\ldots$ \\
\hline pic14 & CF7744514 & $>150$ & 0.08 & $0.01 \pm 0.01 *$ & No hit & $\ldots$ & $\ldots$ \\
\hline pic15 & CF762010 & $>150$ & 0.04 & $0.02 \pm 0.02 *$ & No hit & $\ldots$ & $\ldots$ \\
\hline pic16 & AY093611 & $>150$ & 0.23 & $0.01 \pm 0.01 *$ & AY093611 & Calcium/calmodulin protein kinase, $P$. infestans & 0.0 \\
\hline pic17 & CF798507 & $>150$ & 0.12 & $0.03 \pm 0.01$ & No hit & & $\ldots$ \\
\hline pic18 & CF7744515 & $>150$ & 0.03 & $0.01 \pm 0.01 *$ & AAA31119 & Ryanodine receptor calcium channel, Sus scrofa & $3 \mathrm{E}-17$ \\
\hline pic19 & CF7744516 & $>150$ & 0.02 & $0.01 \pm 0.01 *$ & NP563930 & Phosphatidylinositol kinase, A. thaliana & $2 \mathrm{E}-47$ \\
\hline pic20 & CF7744517 & $137 \pm 20$ & 0.03 & $0.01 \pm 0.01 *$ & No hit & $\ldots$ & $\ldots$ \\
\hline pic21 & CF7744518 & $126 \pm 12$ & 0.11 & $0.00 \pm 0.00$ & No hit & $\ldots$ & $\ldots$ \\
\hline pic22 & CF798508 & $108 \pm 2$ & 0.04 & $0.09 \pm 0.03$ & No hit & $\ldots$ & $\ldots$ \\
\hline pic23 & CF7744519 & $93 \pm 11$ & 0.02 & $0.01 \pm 0.01 *$ & AF466305 & ABC transporter, Dictyostelium discoideum & $1 \mathrm{E}-100$ \\
\hline pic24 & CF798509 & $88 \pm 8$ & 0.09 & $0.02 \pm 0.01$ & No hit & $\ldots$ & $\ldots$ \\
\hline pic25 & CF7744520 & $87 \pm 18$ & 0.01 & $0.02 \pm 0.02 *$ & No hit & $\ldots$ & $\ldots$ \\
\hline pic26 & CF7744521 & $81 \pm 8$ & 0.04 & $0.01 \pm 0.01^{*}$ & No hit & $\ldots$ & $\ldots$ \\
\hline pic27 & CF7744522 & $77 \pm 5$ & 0.01 & $0.01 \pm 0.01 *$ & AAL37628 & $\beta$-Glucanosyltransferase, Coccidioides posadasii & $4 \mathrm{E}-11$ \\
\hline pic 28 & CF7744523 & $70 \pm 2$ & 0.04 & $0.01 \pm 0.01 *$ & No hit & $\ldots$ & $\ldots$ \\
\hline pic29 & CF7744524 & $68 \pm 13$ & 0.06 & $0.01 \pm 0.01 *$ & No hit & $\ldots$ & $\ldots$ \\
\hline pic 30 & CF7744525 & $68 \pm 15$ & 0.05 & $0.02 \pm 0.01$ & No hit & $\ldots$ & $\ldots$ \\
\hline
\end{tabular}

${ }^{\mathrm{a}} \mathrm{CS}=$ cleaving sporangia.

${ }^{\mathrm{b}}$ GenBank accession numbers.

${ }^{\mathrm{c}}$ Ratio of hybridization signal in cleaving sporangia versus uncleaved sporangia, normalized to elongation factor-1 (EF1) level in each sample (i.e., induction ratio). Genes with values $>150$ had no detectable signal using the uncleaved probe. Error (e) in the induction ratio (R) was calculated from replicate measurements in cleaved (c) and uncleaved $(\mathrm{u})$ cDNA hybridizations as $\mathrm{R} \times$ the square root of $\left(\left[\mathrm{e}_{\mathrm{c}} / \mathrm{c}\right]^{2}+\left[\mathrm{e}_{\mathrm{u}} / \mathrm{u}\right]^{2}\right)$.

${ }^{\mathrm{d}}$ Values calculated as being above zero, but not significantly above background, are marked with an asterisk $(*)$.

${ }^{\mathrm{e}}$ BLAST $E$ value. 
Effects of inhibitors on development and gene expression.

The sensitivity of zoosporogenesis and pic expression to various inhibitors was examined to reveal factors regulating those processes, besides cool temperature. The compounds were two calcium channel blockers known "to" inhibit the zoospore pathway, verapamil and gadolinium (Donaldson and Deacon 1993; Hill et al. 1998; Judelson and Roberts 2002; Warburton and Deacon 1998), and two inhibitors of signaling pathways not previously tested in Phytophthora spp. The latter group included 2-aminoethoxydiphenyl borate (2-APB) (Maruyama et al. 1997), which antagonizes inositol trisphosphate $\left(\mathrm{IP}_{3}\right)$ receptor-gated calcium channels (Patel et al. 1999), and U-73122, which inhibits the $\mathrm{IP}_{3}$ generator phospholipase C (Smith et al. 1990).

Each inhibitor blocked zoosporogenesis (Table 2). Except for U-73122, these had little effect on direct germination or hyphal growth. Hill and associates (1998) reported a greater effect of verapamil and gadolinium on direct germination in $P$. parasitica; however, this may be attributed to differences in the media used. That U-73122 but not 2-APB blocked both direct germination and hyphal growth suggests a role of diacylglycerol in those stages. U-73343, an inactive variant of U73122 (Smith et al. 1990), had no effect on growth or development (data not shown).

The inhibitors also affected pic mRNA levels; however, diverse responses were detected. Identical patterns were observed in each of two hybridization experiments, which examined RNA from independent cultures. One experiment is illustrated by the RNA blots in Figure 3A, while both are represented in the quantitations shown in Figure $3 \mathrm{~B}$ (in which a value of 1.0 equals the actin-normalized level in cleaving sporangia without inhibitors; i.e., CS \pm inh "-"”).

The most common class of genes, represented by picl, was sensitive to U-73122 and 2-APB but not verapamil or gadolinium. This implies regulation by $\mathrm{IP}_{3}$ receptor-mediated calcium signaling. A second class, containing pic18, was similar in that RNA accumulation was inhibited by U-73122 and 2-APB; however, verapamil and gadolinium resulted in a twofold increase. Pic18 mRNA normally peaks early after exposing spo-

Table 2. Effect of inhibitors on Phytophthora infestans development ${ }^{\mathrm{a}}$

\begin{tabular}{lccc}
\hline Inhibitor & $\begin{array}{c}\text { Zoospore } \\
\text { release (\%) }\end{array}$ & $\begin{array}{c}\text { Direct } \\
\text { germination (\%) }\end{array}$ & $\begin{array}{c}\text { Hyphal } \\
\text { growth (\%) }\end{array}$ \\
\hline None & 100 & 100 & 100 \\
$10 \mu \mathrm{M} \mathrm{2-APB}$ & $3 \pm 3$ & $103 \pm 14$ & $109 \pm 13$ \\
$200 \mu \mathrm{M}$ verapamil & $10 \pm 7$ & $98 \pm 6$ & $99 \pm 4$ \\
$10 \mu \mathrm{M} \mathrm{GdCl}$ & $10 \pm 9$ & $94 \pm 32$ & $103 \pm 4$ \\
$1 \mu \mathrm{M} \mathrm{U}-73122$ & $3 \pm 3$ & $1 \pm 2$ & $0 \pm 0$ \\
\hline
\end{tabular}

a Sporangia were harvested in water to which the respective inhibitors had been added. To induce zoospore release, the sporangia were incubated for $1 \mathrm{~h}$ at $10^{\circ} \mathrm{C}$ in water and were examined microscopically for the percent releasing zoospores. For direct germination, the sporangia were incubated in rye broth for $24 \mathrm{~h}$ at $18^{\circ} \mathrm{C}$. Values are averages \pm range from three experiments, standardized to $100 \%$ for solvent-only controls.

Table 1. (continued from preceding page)

\begin{tabular}{|c|c|c|c|c|c|c|c|}
\hline \multirow[b]{2}{*}{ Gene } & \multirow[b]{2}{*}{ Number ${ }^{b}$} & \multicolumn{2}{|c|}{ mRNA level in CS vs. ${ }^{a}$} & \multirow{2}{*}{$\begin{array}{c}\text { Hyphae vs. } \\
\text { CS }^{d}\end{array}$} & \multicolumn{3}{|c|}{ Best hit in GenBank } \\
\hline & & Sporangia $^{c}$ & EF-1 & & Number ${ }^{\text {b }}$ & Gene description, source species & $E$ value $^{\mathrm{e}}$ \\
\hline$\overline{p i c 31}$ & CF7744526 & $65 \pm 21$ & 0.02 & $0.03 \pm 0.01$ & Q9EQ15 & Guanine nucleotide-binding protein, $M$. musculus & $2 \mathrm{E}-17$ \\
\hline pic32 & CF7744527 & $62 \pm 5$ & 0.08 & $0.01 \pm 0.01 *$ & No hit & $\ldots$ & $\ldots$ \\
\hline pic33 & CF7744528 & $53 \pm 3$ & 0.05 & $0.05 \pm 0.02$ & XP128418 & Hypothetical protein, $M$. musculus & $2 \mathrm{E}-59$ \\
\hline pic34 & CF7744529 & $49 \pm 3$ & 0.03 & $0.01 \pm 0.00$ & No hit & $\ldots$ & $\ldots$ \\
\hline pic35 & CF7744530 & $45 \pm 2$ & 0.04 & $0.03 \pm 0.01$ & No hit & $\ldots$ & $\ldots$ \\
\hline pic36 & CF7744531 & $44 \pm 1$ & 0.05 & $0.01 \pm 0.01 *$ & NP850136 & Inorganic phosphate transporter, A. thaliana & $2 \mathrm{E}-42$ \\
\hline pic37 & CF7744532 & $43 \pm 7$ & 0.04 & $0.01 \pm 0.01 *$ & No hit & $\ldots$ & $\ldots$ \\
\hline pic38 & CF762011 & $40 \pm 5$ & 0.02 & $0.02 \pm 0.00$ & NP013796 & Phosphatidylinositol transfer protein, Saccharomyces cerevisiae & $3 \mathrm{E}-13$ \\
\hline pic39 & CF7744533 & $39 \pm 3$ & 0.01 & $0.04 \pm 0.02$ & No hit & $\ldots$ & $\ldots$ \\
\hline pic40 & CF7744534 & $39 \pm 5$ & 0.01 & $0.02 \pm 0.01$ & Q13043 & Ste20-like protein kinase, Homo sapiens & $3 \mathrm{E}-79$ \\
\hline pic41 & CF7744535 & $34 \pm 2$ & 0.07 & $0.02 \pm 0.01$ & AAF49704 & Hypothetical protein, Caenorhabditis elegans & $1 \mathrm{E}-05$ \\
\hline pic42 & CF7744536 & $30 \pm 2$ & 0.04 & $0.02 \pm 0.01$ & No hit & $\ldots$ & $\ldots$ \\
\hline pic43 & CF7744537 & $29 \pm 1$ & 0.04 & $0.04 \pm 0.02$ & P26414 & Endoglucanase, Thermobispora bispora & $1 \mathrm{E}-24$ \\
\hline pic44 & CF7744538 & $28 \pm 3$ & 0.02 & $0.10 \pm 0.02$ & No hit & $\ldots$ & $\ldots$ \\
\hline pic45 & CF7744539 & $28 \pm 0$ & 0.01 & $0.04 \pm 0.01$ & No hit & $\ldots$ & $\ldots$ \\
\hline pic46 & CF7744540 & $28 \pm 2$ & 0.06 & $0.05 \pm 0.01$ & No hit & $\ldots$ & $\ldots$ \\
\hline pic47 & CF7744541 & $27 \pm 2$ & 0.04 & $0.09 \pm 0.01$ & AAB08700 & Ubiquitin-conjugating enzyme, Dictyostelium discoideum & $3 \mathrm{E}-39$ \\
\hline pic48 & CF7744542 & $26 \pm 2$ & 0.01 & $0.04 \pm 0.02$ & No hit & $\ldots$ & $\ldots$ \\
\hline pic49 & CF7744543 & $24 \pm 2$ & 0.05 & $0.02 \pm 0.01$ & No hit & $\ldots$ & $\ldots$ \\
\hline pic50 & CF7744544 & $24 \pm 1$ & 0.04 & $0.06 \pm 0.03$ & P31320 & cAMP-dependent kinase subunit, Blastocladiella emersonii & $1 \mathrm{E}-47$ \\
\hline pic51 & AF507056 & $23 \pm 6$ & 0.01 & $0.06 \pm 0.02$ & AF507056 & Transglutaminase, $P$. infestans & 0 \\
\hline pic52 & CF7744542 & $20 \pm 1$ & 0.04 & $0.04 \pm 0.01$ & No hit & $\ldots$ & $\ldots$ \\
\hline pic53 & CF7744546 & $20 \pm 0$ & 0.11 & $0.07 \pm 0.01$ & No hit & $\ldots$ & $\ldots$ \\
\hline pic54 & CF7744547 & $19 \pm 1$ & 0.17 & $0.06 \pm 0.01$ & No hit & $\ldots$ & $\ldots$ \\
\hline pic55 & CF7744548 & $18 \pm 3$ & 0.01 & $0.06 \pm 0.03$ & NP594488 & RNA helicase, Schizosaccharomyces pombe & $2 \mathrm{E}-46$ \\
\hline pic56 & CF7744549 & $18 \pm 1$ & 0.05 & $0.04 \pm 0.01$ & NP596878 & Trimethyllysine hydroxylase, Rattus norvegicus & $1 \mathrm{E}-46$ \\
\hline pic57 & CF7744550 & $14 \pm 2$ & 0.05 & $0.07 \pm 0.02$ & No hit & $\ldots$ & $\ldots$ \\
\hline pic58 & CF7744551 & $14 \pm 2$ & 0.16 & $0.04 \pm 0.01$ & NP181121 & Serine carboxypeptidase, A. thaliana & $3 \mathrm{E}-76$ \\
\hline pic59 & CF7744552 & $12 \pm 2$ & 0.02 & $0.10 \pm 0.04$ & AAD11618 & P-type calcium-ATPase, Plasmodium falciparum & $2 \mathrm{E}-64$ \\
\hline pic60 & CF7744553 & $10 \pm 0$ & 0.05 & $0.11 \pm 0.03$ & No hit & $\ldots$ & $\ldots$ \\
\hline pic61 & CF762007 & $9 \pm 1$ & 0.05 & $0.10 \pm 0.01$ & NP506621 & Carnitine acylcarnitine translocase, Caenorhabditis elegans & $2 \mathrm{E}-28$ \\
\hline pic62 & CF762008 & $8 \pm 0$ & 0.01 & $0.17 \pm 0.06$ & No hit & $\ldots$ & $\ldots$ \\
\hline pic63 & CF7744554 & $6 \pm 2$ & 0.01 & $0.13 \pm 0.13$ & AAK 31375 & Proteophosphoglycan, Leishmania major & $1 \mathrm{E}-07$ \\
\hline pic64 & CF7744555 & $6 \pm 1$ & 0.03 & $0.22 \pm 0.07$ & NP195754 & Mitochondrial citrate transport protein, A. thaliana & $1 \mathrm{E}-68$ \\
\hline pic65 & CF7744556 & $5 \pm 0$ & 0.01 & $0.06 \pm 0.01$ & AA052242 & Hypothetical protein, Dictyostelium discoideum & $6 \mathrm{E}-13$ \\
\hline pic66 & CF7744557 & $5 \pm 0$ & 0.06 & $0.09 \pm 0.01$ & AAL05057 & Ribonucleotide reductase, Aedes aegypti & $1 \mathrm{E}-123$ \\
\hline pic67 & CF762009 & $4 \pm 0$ & 0.01 & $0.16 \pm 0.02$ & No hit & $\ldots$ & $\ldots$ \\
\hline pic68 & CF7744558 & $4 \pm 1$ & 0.01 & $0.12 \pm 0.07$ & No hit & $\ldots$ & $\ldots$ \\
\hline pic69 & CF7744559 & $4 \pm 1$ & 0.01 & $0.20 \pm 0.12$ & No hit & $\ldots$ & $\ldots$ \\
\hline
\end{tabular}


rangia to cool water and then falls, suggesting that verapamil and gadolinium block factors that normally turn off its transcription or hasten degradation of its mRNA midway through cleavage. A third class of genes, typified by pic16, was repressed only by U-73122. Because mRNA accumulation was not blocked by 2-APB, the inhibitor of the target of $\mathrm{IP}_{3}$, diacylglycerol may be implicated in regulating the expression of such genes. A fourth class of genes, exemplified by pic29, was repressed by each inhibitor. This may suggest regulation by $\mathrm{Ca}^{2+}$ influx through the $\mathrm{IP}_{3}$ receptor and other channels. A fifth class of genes, represented by pic 27, was not significantly blocked by either inhibitor. Considering that pic27 mRNA peaks sooner than others during cleavage (Fig. 2A), either a distinct pathway regulates pic 27 or the inhibitors did not penetrate sporangia fast enough to block its expression.

\section{DISCUSSION}

Zoosporogenesis is a remarkable developmental process, during which cold triggers a rapid reorganization of the contents of sporangia. To help understand this pathway, we have focused on identifying genes that exhibit a substantial increase in mRNA abundance during cleavage, although genes exhibiting more subtle changes also may be important. Our results indicate that the transcriptional profile of sporangia also is altered by cold, inducing at least 20 genes not expressed in other developmental stages and up-regulating at least 50 others. More zoosporogenesis-induced genes likely exist, but their discovery must wait until a complete annotated genome sequence is available for $P$. infestans; although all of the estimated 2,617 genes assayed by our arrays were from a cleaving cDNA library, many weakly transcribed genes probably were missed. However, it is notable that the percentage of genes specific to cleavage $(0.8 \%)$ is remarkably close to the fraction of protein spots $(0.9 \%)$ reported to be cleavage or zoospore specific in a recent two-dimensional protein gel study of Phytophthora spp. development (Shepherd et al. 2003).

A large fraction of the pic genes (66\%) lacked homologues in other organisms, but this is expected, considering that oomycetes are taxonomically dissimilar to well-studied species, including true fungi (Gunderson et al. 1987) and the relative novelty of zoospores. Nevertheless, the fraction of pic sequences resembling known genes was much less than detected in other gene discovery projects in Phytophthora spp., which demonstrates the novelty of the zoospore pathway.

The pic genes became up-regulated within $20 \mathrm{~min}$ of exposure to cold temperatures, well before cleavage and zoospore liberation. However, it does not follow that zoospore formation requires these genes, considering reports that actinomycin $\mathrm{D}$ fails to block zoospore release or encystment (Clark et al. 1978; Penington et al. 1989). It may be more appropriate to consider pic genes as participants in subsequent stages such as cyst germination or appressorium formation.

Although the pic genes may be unneeded for cleavage, they furnish a valuable tool for studying signaling during that stage. Prior to identifying the genes, we developed a simple model to explain how placing sporangia in cool water leads to zoospores. In that scheme, immersion either releases a germination inhibitor or hydrates a receptor on sporangial surfaces. Chilling then reduces fluidity of the plasma membrane, which either directly activates phospholipase $\mathrm{C}$ or modifies the interaction between phospholipase $\mathrm{C}$ and another membrane-bound protein.; similar events have been invoked to explain chilling responses in plants (Bigot and Boucaud 2000; Suh et al. 2001). Phospholipase $C$ then would lead to the activation of an $\mathrm{IP}_{3}$ receptor-gated calcium channel, which would increase cytoplasmic calcium levels, and stimulate calcium or calmo- dium kinases or phosphodiesterases. This ultimately would result in the cytoplasmic rearrangements that characterize cleavage and the activation of transcription factors needed to synthesize proteins required for later stages such as cyst germination.

The integration of a germination inhibitor into the above model is necessary to explain why zoosporogenesis requires both cool temperatures and immersion in water. Unlike conidia of true fungi, oomycete sporangia remain fully hydrated and even slight desiccation destroys viability (Cohen et al. 1974). It is possible in $P$. infestans that contact with water releases an inhibitor of phospholipase $\mathrm{C}$ or another regulator such as an ion channel. Ion transport has been shown to be blocked by selfinhibitors in other fungi (Breeuwer et al. 1997).

Our observations that 2-APB and U-73122 block zoosporogenesis are consistent with the above model, but the diverse effects of these and other inhibitors on the pic genes suggest that a more complex scheme for signal transduction and differentiation is required. The varied kinetics of accumulation of different pic genes also leads to the same conclusion. The detection of pic genes sensitive and insensitive to either 2-APB or U-73122, for example, suggests the involvement of signaling molecules which remain to be identified. Although phospholipids besides $\mathrm{IP}_{3}$, such as phosphatidic acid, are implicated in zoospore encystment, their participation in cleavage has not been demonstrated (Latijnhouwers et al. 2002; Zhang et al. 1992). Attempts to implicate changes in the levels of cyclic nucleotides to Phytophthora spp. development also have yielded negative results (Bircher and Hohl 1999; Zhang et al. 1992). However, oscillating concentrations of signaling molecules rather than a prolonged increase may regulate cleavage, as observed for $\mathrm{IP}_{3}$ in other systems (Falcke 2003). Our laboratory has confirmed that $P$. infestans sporangia contain $\mathrm{IP}_{3}$, but failed to demonstrate a convincing increase in its concentration during cleavage (unpublished).

Inhibitors of these signaling molecules did not suppress the levels of most pic mRNAs to the same degree that they inhibited zoospore release. This may be explained if the inhibitors entered sporangia too slowly to block the initial burst of expression of these genes. Alternatively, transcription and zoosporogenesis per se may involve different targets of the inhibitors, or zoosporogenesis may be more sensitive to the additive effects of partially inhibiting several cellular processes.

Although more work is required to understand how signals from different secondary messengers are integrated to regulate zoospore development, several of the predicted PIC proteins appear to have regulatory functions that may be important in the zoospore pathway. These include a putative $G$ protein (PIC31), a transcriptional coactivator (PIC1), two ion transporters (PIC18 and PIC59), and three protein kinases (PIC16, PIC40, and PIC50). Although their functions need to be confirmed biochemically, based on sequence similarity, two of the kinases may interact with secondary messengers (cAMP or calcium) and two of the transporters interact with calcium. Such proteins may provide further means to understand the relationship between secondary messengers and cellular events during Phytophthora spp. development.

\section{MATERIALS AND METHODS}

\section{Manipulations of $P$. infestans.}

Isolate 1306 (A1 mating type, USA) was maintained at $18^{\circ} \mathrm{C}$ on rye A agar or clarified rye both, and manipulated to induce developmental stages essentially as described (Judelson and Roberts 2002). Briefly, sporangia were liberated from 9to 13-day-old rye agar cultures by agitation in cool water, and separated from hyphal fragments by passage through $50-\mu \mathrm{m}$ 
nylon mesh. Sporangia $\left(10^{5} / \mathrm{ml}\right)$ were placed in $24^{\circ} \mathrm{C}$ water for the times indicated above to induce direct germination, or at $10^{\circ} \mathrm{C}$ for the times indicated above to induce zoospores. The latter involved placing samples on ice for several minutes to quickly cool them to $10^{\circ} \mathrm{C}$, followed by placement in a $10^{\circ} \mathrm{C}$ incubator. Germinated cysts were made from zoospores that were vortexed for $1 \mathrm{~min}$ and then incubated at $10^{\circ} \mathrm{C}$ for $6 \mathrm{~h}$. RNA was extracted from each developmental stage by guanidium hydrochloride extraction (Judelson and Roberts 2002).

\section{cDNA array and RNA blot analysis.}

A cDNA library generated from cleaving sporangia of isolate 88069 (A1, The Netherlands) was used as the source of DNA for array construction. The library previously had been subjected to $5^{\prime}$ single-pass sequencing as part of an EST project (Lam 2001). Inserts were amplified by two-step PCR using SPORTL (5'-ACGTCGCATGCACGCGTACGTAAGC) and SPORTR (5'-ACGACTCACTATAGGGAAAGCTGGTACG), a $94^{\circ} \mathrm{C}$ denaturation step, and a $72^{\circ} \mathrm{C}$ annealing or extension phase. A fraction of the reaction products were checked on a gel for purity and concentration, and then spotted on nylon membranes using a 96 -well tool delivering $0.5 \mu \mathrm{l}$ per pin ( $\mathrm{V}$ and P Scientific, San Diego, CA). Also spotted were actin (actA) and EF1 clones for normalization, and controls for nonspecific hybridization. Arrays were hybridized with ${ }^{32} \mathrm{P}$-labeled cDNA from sporangia, cleaving sporangia $\left(40 \mathrm{~min}\right.$ at $10^{\circ} \mathrm{C}$ ), and hyphae as described (Fabritius et al. 2002). Primary screens were performed using replicate filters, with each cDNA clone represented once. Secondary and tertiary screens included replicates of each clone. Spotted DNA was quantified by hybridizations with end-labeled SPORTL. Signals were quantified by phosphorimager analysis using Quantity One for Macintosh (Biorad, Richmond, CA, U.S.A.). After adjustments for background, data were filtered based on the signal-to-noise ratio and analyzed for significant differences in mRNA abundance in developmental stages by a Student's $t$ test.

Blots of total mRNA ( $5 \mu \mathrm{g}$ per lane) were prepared using formaldehyde gels and hybridized as described (Judelson and Roberts 2002). Signals were quantified by phosphorimager analysis.

\section{Sequence analysis.}

cDNA clones showing differential expression were subjected to $5^{\prime}$ and $3^{\prime}$ sequencing. The resulting sequences were trimmed of vector and assembled using Seqman for Macintosh (DNAStar, Madison, WI, U.S.A.) and compared with sequences in GenBank by BLASTX using a server in the campus core genomics facility.

\section{ACKNOWLEDGMENTS}

This work was supported by awards from the United States Department of Agriculture, Syngenta Corporation, and the Industry-University Research Competitive Grants Program of the University of California. We thank T. Randall for assisting in the curation of cDNA clones, S. Roberts for technical assistance, and $\mathrm{L}$. Vaillancort for a gift of $\mathrm{GdCl}_{3}$.

\section{LITERATURE CITED}

Bigot, J., and Boucaud, J. 2000. Effects of ca-signalling inhibitors on short-term cold-acclimation of hydraulic conductivity in roots of Brassica rapa plants. J. Plant Physiol. 157:7-12.

Bircher, U., and Hohl, H. R. 1999. A role for calcium in appressorium induction in Phytophthora palmivora. Bot. Helv. 109:55-65.

Bockaert, J., and Pin, J. P. 1999. Molecular tinkering of G protein-coupled receptors: An evolutionary success. EMBO (Eur. Mol. Biol. Organ.) J. 18:1723-1729.
Breeuwer, P., De Reu Johan, C., Drocourt, J.-L., Rombouts Frank, M., and Abee, T. 1997. Nonanoic acid, a fungal self-inhibitor, prevents germination of Rhizopus oligosporus sporangiospores by dissipation of the ph gradient. Appl. Environ. Microbiol. 63:178-185.

Brunner, F., Rosahl, S., Lee, J., Rudd, J. J., Geiler, C., Kauppinen, S., Rasmussen, G., Scheel, D., and Nuernberger, T. 2002. Pep-13, a plant defense-inducing pathogen-associated pattern from Phytophthora transglutaminases. EMBO (Eur. Mol. Biol. Organ.) J. 21:6681-6688.

Clark, M. C., Melanson, D. L., and Page, O. T. 1978. Purine metabolism and differential inhibition of spore germination in Phytophthora infestans. Can. J. Microbiol. 24:1032-1038.

Cohen, Y., Perl, M., Rotem, J., Eyal, H., and Cohen, J. 1974. Ultrastructural and physiological changes in sporangia of Pseudoperonospora cubensis and Phytophthora infestans exposed to water stress. Can. J. Bot. 52:447-450.

Donaldson, S. P., and Deacon, J. W. 1993. Changes in motility of Pythium zoospores induced by calcium and calcium-modulating drugs. Mycol. Res. 97:877-883.

Duniway, J. M. 1983. Role of physical factors in the development of Phytophthora diseases. Pages 175 to 187 in: Phytophthora: Its Biology, Taxonomy, Ecology, and Pathology. D. C. Erwin, S. Bartnicki-Garcia, and P. H. Tsao, eds. American Phytopathological Society Press, St. Paul, MN, U.S.A.

Fabritius, A.-L., and Judelson, H. S. 2003. A mating-induced protein of Phytophthora infestans is a member of a family of elicitors with divergent structures and stage-specific patterns of expression. Mol. PlantMicrobe Interact.16:926-935.

Falcke, M. 2003. On the role of stochastic channel behavior in intracellular $\mathrm{Ca}^{2+}$ dynamics. Biophys. J. 84:42-56.

Gaffin, S., L. 1999. Simplified calcium transport and storage pathways. J. Therm. Biol. 24:251-264.

Griffith, J. M., Iser, J. R., and Grant, B. R. 1988. Calcium control of differentiation in Phytophthora palmivora. Arch. Microbiol. 149:565571.

Gunderson, J. H., Elwood, H., Ingold, A., Kindle, K., and Sogin, M. L. 1987. Phylogenetic relationships between chlorophytes, chrysophytes, and oomycetes. Proc. Nat. Acad. Sci. U.S.A. 84:5823-5827.

Hardham, A. R. 2001. The cell biology behind Phytophthora pathogenicity. Aust. Plant Pathol. 30:91-98.

Hill, A. E., Grayson, D. E., and Deacon, J. W. 1998. Suppressed germination and early death of Phytophthora infestans sporangia caused by pectin, inorganic phosphate, ion chelators and calcium-modulating treatments. Eur. J. Plant Pathol. 104:367-376.

Jackson, S. L., and Hardham, A. R. 1996. A transient rise in cytoplasmic free calcium is required to induce cytokinesis in zoosporangia of $P h y$ tophthora cinnamomi. Eur. J. Cell Biol. 69:180-188.

Judelson, H. S., and Roberts, S. 2002. Novel protein kinase induced during sporangial cleavage in the oomycete Phytophthora infestans. Eukaryotic Cell 1:687-695.

Kamoun, S., Hraber, P., Sobral, B., Nuss, D., and Govers, F. 1999. Initial assessment of gene diversity for the oomycete pathogen Phytophthora infestans based on expressed sequences. Fungal Genet. Biol. 28:94106.

Kim, K. S., and Judelson, H. S. 2003. Sporangia-specific gene expression in the oomycete phytopathogen Phytophthora infestans. Eukaryotic Cell 2:1376-1385.

Lam, S. T. 2001. Phytophthora genomics consortium. Phytopathology 91:S158.

Latijnhouwers, M., Munnik, T., and Govers, F. 2002. Phospholipase D in Phytophthora infestans and its role in zoospore encystment. Mol. PlantMicrobe Interact. 15:939-946.

Marshall, J. S., Wilkinson, J. M., Moore, T., and Hardham, A. R. 2001. Structure and expression of the genes encoding proteins resident in large peripheral vesicles of Phytophthora cinnamomi zoospores. Protoplasma 215:226-239.

Maruyama, T., Kanaji, T., Nakade, S., Kanno, T., and Mikoshiba, K. 1997. 2APB, 2-aminoethoxydiphenyl borate, a membrane-penetrable modulator of ins $(1,4,5) \mathrm{p}-3$-induced $\mathrm{Ca}^{2+}$ release. J. Biochem. 122:498-505.

Patel, S., Joseph, S. K., and Thomas, A. P. 1999. Molecular properties of inositol 1,4,5-trisphosphate receptors. Cell Calcium 25:247-264.

Penington, C. J., Iser, J. R., Grant, B. R., and Gayler, K. R. 1989. Role of RNA and protein synthesis in stimulated germination of zoospores of the pathogenic fungus Phytophthora palmivora. Exp. Mycol. 13:158168.

Ribeiro, O. K. 1983. Physiology of asexual sporulation and spore germination in Phytophthora. Pages 55-70 in: Phytophthora: Its Biology, Taxonomy, Ecology, and Pathology. D. C. Erwin, S. Bartnicki-Garcia, and P. H. Tsao, eds. American Phytopathological Society Press, St. Paul, MN, U.S.A. 
Shepherd, S. J., Van West, P., and Gow, N. A. R. 2003. Proteomic analysis of asexual development of Phytophthora palmivora. Mycol. Res. 107:395-400.

Smith, R. J., Sam, L. M., Justen, J. M., Bundy, G. L., Bala, G. A., and Bleasdale, J. E. 1990. Receptor-coupled signal transduction in human polymorphonuclear neutrophils effects of a novel inhibitor of Phospholipase C-dependent processes on cell responsiveness. J. Pharmacol. Exp. Ther. 253:688-697.

Suh, P.-G., Hwang, J.-I., Ryu, S. H., Donowitz, M., and Kim, J. H. 2001. The roles of PDZ-containing proteins in PLC-beta-mediated signaling.
Biochem. Biophys. Res. Commun. 288:1-7.

Taniguchi, M., Miura, K., Iwao, H., and Yamanaka, S. 2001. Quantitative assessment of DNA microarrays: Comparison with Northern blot analyses. Genomics 71:34-39.

Warburton, A. J., and Deacon, J. W. 1998. Transmembrane $\mathrm{Ca}^{2+}$ fluxes associated with zoospore encystment and cyst germination by the phytopathogen Phytophthora parasitica. Fungal Gen. Biol. 25:54-62.

Zhang, Q., Griffith, J. M., and Grant, B. R. 1992. Role of phosphatidic acid during differentiation of Phytophthora palmivora zoospores. J. Gen. Microbiol. 138:451-459. 